\title{
Are Higher Education Institutions Addressing the Employment Needs of Clinical Laboratories in Rural Areas?
}

Clinical laboratories across the nation are faced with a shortage of qualified Medical Laboratory

Technicians and Medical Laboratory Scientists. As the clinical laboratory workforce continues to shrink, it is essential that higher education institutions acknowledge the employment needs of rural laboratories to ensure quality health care in underserved areas. An aging workforce, program closures, and a general increase in demand for laboratory testing have compounded the workforce shortage for rural clinical laboratories, which often face unique challenges. The purpose of this research study was to examine the perceptions of rural clinical laboratory managers toward higher education's response to the shortage of MLTs and MLSs, and to determine the extent to which MLT and MLS programs are meeting the staffing and professional development needs of rural clinical laboratories. This descriptive mixed-method study was conducted utilizing a national survey of 429 MLT and MLS program directors, in addition to interviews with 10 clinical laboratory managers in rural West Virginia. The results of this study revealed higher education institutions are not adequately meeting the employment needs of rural clinical laboratories, with notable key areas for improvement, including communication, partnership, and promotion of the profession. Interviews with laboratory managers indicated a moderate to severe rural workforce shortage, with a glaring deficiency of MLS candidates. Research findings demonstrated that community and technical colleges, specifically MLT programs, were more likely than universities and four-year colleges to establish formal relationships with rural laboratories and served as a primary source of job candidates for rural laboratories. The findings further suggested rural clinical rotations, targeted recruitment efforts, and the creation of online and hybrid programs, would aid rural laboratories in securing a 
qualified workforce, while the delivery of online continuing education modules would benefit incumbent workers. 